\title{
Chordal Graphs as Intersection Graphs of Pseudosegments
}

\author{
Cornelia Dangelmayr ${ }^{1}$ and Stefan Felsner ${ }^{2}$ \\ ${ }^{1}$ Freie Universität Berlin \\ Institut für Mathematik II, \\ Arnimallee 3, \\ 14195 Berlin, Germany \\ dangel@math.fu-berlin.de \\ 2 Technische Universität Berlin \\ Institut für Mathematik, MA 6-1 \\ Strasse des 17. Juni 136 \\ 10623 Berlin, Germany \\ felsner@math.tu-berlin.de
}

\begin{abstract}
We investigate which chordal graphs have a representation as intersection graphs of pseudosegments. The main contribution is a construction which shows that all chordal graphs which have a representation as intersection graph of subpaths on a tree are representable. A family of intersection graphs of substars of a star is used to show that not all chordal graphs are representable by pseudosegments.
\end{abstract}

\section{Introduction}

A set of Jordan arcs induces a graph $G$, the vertices of $G$ are the Jordan arcs and two Jordan arcs are adjacent if and only if they have non-empty intersection. Graphs representable in this model are called string graphs. String graphs are quite complicated, only recently it has been shown that membership in this class is at least decidable [7/11]. All planar graphs are string graphs, this follows e.g. from Koebe's circle representations.

If any two Jordan arcs in a set of nontagent Jordan arcs are either disjoint or have exactly one point of intersection, we call this as set of pseudosegments and the resulting class of graphs as intersection graphs of pseudosegments. We denote this class by PSI. Deciding whether a given graph is a PSI-graph is known to be NP-complete 8]. A main open problem is whether all planar graphs belong to PSI (see [1/2|3|4|5]). Actually, most of the cited references discuss the class of segment intersection graphs, the problem whether all planar graphs are representable is open with respect to this class as well.

There are some classes of graphs where PSI-representations are trivial (e.g. permutation graphs) or very easy to find (e.g. interval graphs). A large superclass of interval graphs is the class of chordal graphs. In this paper we investigate chordal graphs in view of their representability as intersection graphs of pseudosegments. 


\section{Basic Definitions and Results}

Definition 1. A graph $G=\left(V_{G}, E_{G}\right)$ is a VPT-graph 1 , if there exists a tree $T=\left(V_{T}, E_{T}\right)$ and a set $\mathcal{P}$ of paths in $T$ such that there is a mapping $v \rightarrow P_{v} \in \mathcal{P}$ with the property that $v w \in E_{G}$ iff $P_{v} \cap P_{w} \neq \emptyset$. Such a pair $(T, \mathcal{P})$ is said to be a VPT-representation of $G$.

These graphs have been introduced by Gavril [6] who gave a recognition algorithm and have been studied continuously since then. Monma and Wei [10] give some applications and many references. Our first result is:

Theorem 1. Every VPT-graph has a PSI-representation.

The proof of the theorem is given in Section 3. At this point we content ourselves with an indication that the result is not as trivial as it may seem at first glance. Let a VPT-representation $(T, \mathcal{P})$ of a graph $G$ be given. If we fix a plane embedding of the tree we obtain an embedding of each of the paths $P_{v}$ corresponding to $v \in V_{G}$, this embedded path $P_{v}$ is a Jordan arc. The first idea for converting a VPT-representation into a PSI-representation would be to slightly perturb $P_{v}$ into a pseudosegment $s_{v}$ and make sure that paths with common vertices intersect exactly once and are disjoint otherwise. Figure 1 gives an example of a set of subpaths which can't be perturbed such that they give a PSI-representation of the corresponding subgraph.

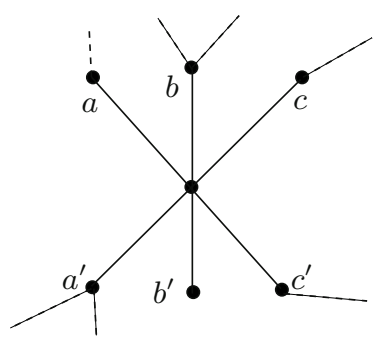

Fig. 1. The paths $P\left(a, a^{\prime}\right), P\left(b, b^{\prime}\right)$ and $P\left(c, c^{\prime}\right)$ can not locally be perturbed into a PSI-representation

A superclass of VPT-graphs is the class of vertex intersection graphs of subtrees of a tree. Graphs with such a representation are exactly the chordal graphs.

Clearly, every cycle $C_{n}, n \in \mathbb{N}$ has a representation as intersection graph of pseudosegments, whereas $C_{n}$ is not chordal for $n \geq 4$. Hence the class PSI is not contained in the class of chordal graphs.

Theorem 2. There are chordal graphs that are not in the class PSI.

The proof of the theorem is given in Section 4. There we use geometric arguments to show that the graph $K_{n}^{3}$ defined below is not in PSI.

\footnotetext{
${ }^{1}$ VPT is mnemonic for vertex intersection graph of paths on a tree.
} 
$K_{n}^{3}$ has two groups of vertices, vertices $v_{1}, . ., v_{n}$ that are a clique and a vertex $v_{i, j, k}$ for every triple $\{i, j, k\} \subset[n]$. These triple-vertices are adjacent only to the three corresponding vertices $v_{i}, v_{j}$ and $v_{k}$, hence form an independent set. The graph $K_{n}^{3}$ can be represented as intersection graph of subtrees of a star $S$ with $\left(\begin{array}{l}n \\ 3\end{array}\right)$ leaves. The leaves correspond to the vertices $v_{i, j, k}$ and these vertices are represented by trivial trees with only one node. A vertex $v_{i}$ of the complete graph is represented by the star connecting to all leaves of triples containing $i$. This representation shows that $K_{n}^{3}$ is chordal.

The central node of the star $S$ has high degree. If we take a path of $\left(\begin{array}{l}n \\ 3\end{array}\right)$ nodes and attach a leaf-node to each node of the path we obtain a tree $T$ of maximum degree three such that the graph $K_{n}^{3}$ can be represented as intersection graph of subtrees of $T$. Actually the tree $T$ and its subtrees are caterpillars of maximum degree three.

These remarks show that the positive result of Theorem 1 and the negative of Theorem 2 only leave a small gap for questions: If the subtrees in a tree representation of a chordal graph are paths we have a PSI-representation. If we allow the subtrees to be stars, or caterpillars of maximum degree three, there need not exist a PSI-representation. We don't know the answer if the subtrees in the representation only have a small constant number of leaves. When the number of leaves is allowed to get as big as $741=\left(\begin{array}{c}39 \\ 2\end{array}\right)$ the graph $K_{39}^{3}$ again shows that the graphs are not in PSI. We think that one node of degree three in each subtree of a tree representation is sufficient to get graphs which are not PSI-representable. More precisely, let $S_{n}$ be the chordal graph whose vertices are represented by all substars with three leaves and all leaves on a star with $n$ leaves.

Conjecture 1. For $n$ large enough, $S_{n}$ is not a PSI-graph.

\section{Proof of Theorem 1}

\subsection{Preliminaries}

Let $P$ be a path in a tree $T$ with endpoints $a$ and $b$, this is denoted $P=P(a, b)$. If both endpoints of $P$ are leaves of $T$ we call $P$ a leaf-path.

Lemma 1. Every VPT-graph has a representation $(T, \mathcal{P})$ such that all paths in $\mathcal{P}$ are leaf-paths and no two vertices are represented by the same leaf-path.

Proof. Let an arbitrary VPT-representation $(T, \mathcal{P})$ of $G$ with $P_{v}=P\left(a_{v}, b_{v}\right)$ for all $v \in V_{G}$ be given. Now let $\bar{T}$ be the tree obtained from $T$ by attaching a new node $\bar{x}$ to every node $x$ of $T$. Representing the vertex $v$ by the path $\overline{P_{v}}=P\left(\overline{a_{v}}, \overline{b_{v}}\right)$ in $\bar{T}$ yields a VPT-representation of $G$ using only leaf-paths.

The definition of VPT-graphs as intersection graphs immediately implies that every induced subgraph of a VPT-graph is a VPT-graph as well. The lemma together with this observation shows that Theorem 1 is implied by the following:

Theorem 3. Given a tree $T$ we let $G$ be the VPT-graph whose vertices are in bijection to the set of all leaf-paths of $T$. The graph $G$ has a PSI-representation 
with pseudosegments $s_{i, j}$ corresponding to the paths $P_{i, j}=P\left(l_{i}, l_{j}\right)$ in $T$. In addition there is a collection of pairwise disjoint disks, one disk $R_{i}$ associated with each leaf $l_{i}$ of $T$, such that:

(a) The intersection $s_{i, j} \cap R_{k} \neq \emptyset$ if and only if $k=i$ or $k=j$. Furthermore the intersections $s_{i, j} \cap R_{i}$ and $s_{i, j} \cap R_{j}$ are Jordan curves.

(b) Any two pseudosegments intersecting $R_{i}$ cross in the interior of this disk.

We will prove Theorem 3 by induction on the number of inner nodes of tree $T$. The construction will have multiple intersections, i.e., there are points where more than two pseudosegments intersect. By perturbing the pseudosegments participating in a multiple intersection locally the representation can easily be transformed into a representation without multiple intersections.

\subsection{Theorem 3 Is True for Trees with One Inner Node}

Let $T$ have one inner node $v$ and let $L=\left\{l_{1}, \ldots, l_{m}\right\}$ be the set of leaves of $T$. The subgraph $H$ of $G$ induced by the set $\mathcal{P}=\left\{P\left(l_{i}, l_{j}\right) \mid l_{i}, l_{j} \in L, l_{i} \neq l_{j}\right\}$ of leaf-paths is a complete graph on $\left(\begin{array}{c}m \\ 2\end{array}\right)$ vertices, this is because every path in $\mathcal{P}$ contains $v$.

Take a circle $\gamma$ and choose $m$ points $c_{1}, \ldots, c_{m}$ on $\gamma$ such that the set of straight lines spanned by pairs of different points from $c_{1}, . ., c_{m}$ contains no parallel lines. For each $i$ choose a small disk $R_{i}$ centered at $c_{i}$ such that these disks are disjoint and put them in one-to-one correspondence with the leaves of $T$. Let $s_{i, j}$ be the line connecting $c_{i}$ and $c_{j}$. If the disks $R_{k}$ are small enough we clearly have :

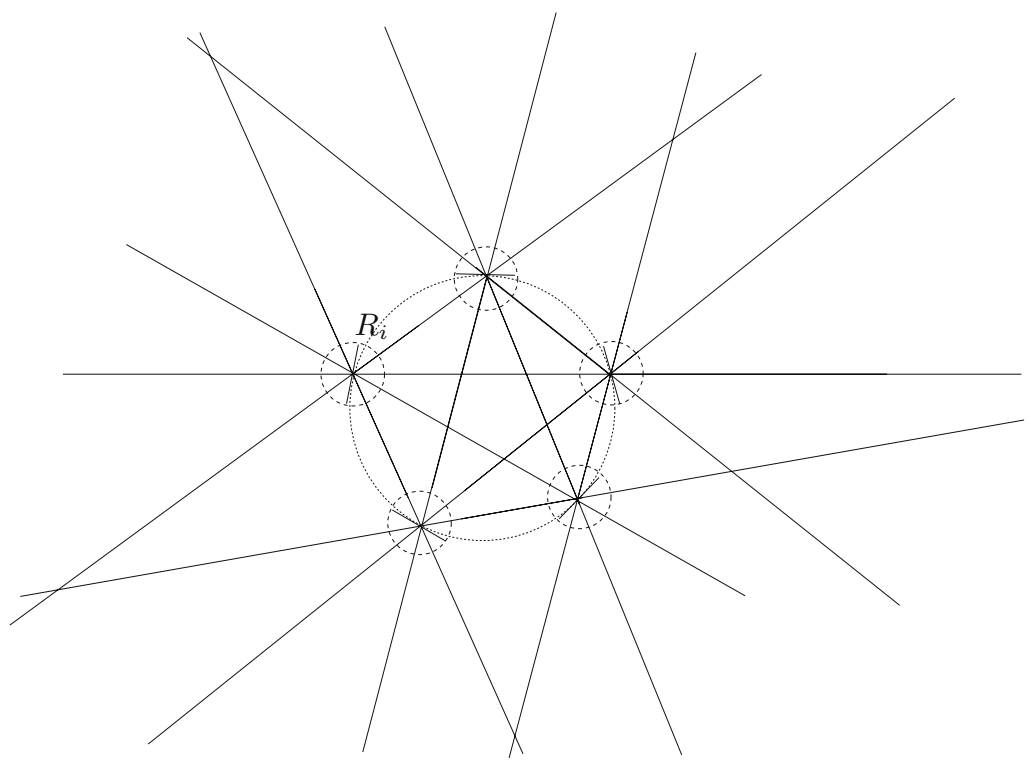

Fig. 2. The construction for the star with five leaves 
(a) The line $s_{i, j}$ intersects $R_{i}$ and $R_{j}$ but no further disk $R_{k}$.

(b) Two lines $s_{I}$ and $s_{J}$ with $I \cap J=\{i\}$ contain corner $c_{i}$, hence $s_{I}$ and $s_{J}$ cross in the disk $R_{i}$.

Prune the lines such that the remaining part of each $s_{i, j}$ still contains its intersection with all the other lines and all segments have there endpoints on a circumscribing circle $C$. Every pair of segments stays intersecting, hence, we have a segment intersection representation of $H$.

Add a diameter $s_{i, i}$ to every disk $R_{i}$, this segment serves as representation for the leaf-path $P_{i, i}$. Altogether we have constructed a representation of $G$ obeying the required properties (a) and (b), see Figure 2 for an illustration.

\subsection{Theorem 3 Is True for Trees with More Than One Inner Node}

Now let $T$ be a tree with inner nodes $N=\left\{v_{1}, . ., v_{n}\right\}$ and assume the Theorem has been proven for trees with at most $n-1$ inner nodes. Let $L=\left\{l_{1}, . ., l_{m}\right\}$ be the set of leaves of $T$. With $L_{i} \subset L$ we denote the set of leaves attached to $v_{i}$. We have to produce a PSI-representation of the intersection graph $G$ of $\mathcal{P}=\left\{P_{i, j} \mid l_{i}, l_{j} \in L\right\}$, i.e., of the set of all leaf-paths of $T$. Let $v_{1}$ be the root of $T, h$ its resulting height and choose a vertex of $N$ with distance $h-1$ from the root, say $v_{n}$. We define two induced subtrees of $T$ :

- The tree $T_{n}$ is the star with inner node $v_{n}$ and its leaves $L_{n}=\left\{l_{k}, . ., l_{m}\right\}$.

- The tree $T^{\prime}$ contains all nodes of $T$ except the leaves in $L_{n}$. The set of inner nodes of $T^{\prime}$ is $N^{\prime}=N \backslash\left\{v_{n}\right\}$, the set of leaves is $L^{\prime}=L \backslash L_{n} \cup\left\{v_{n}\right\}$. For consistency we rename $l_{0}:=v_{n}$ in $T^{\prime}$, hence $L^{\prime}=\left\{l_{0}, l_{1}, . ., l_{k-1}\right\}$.
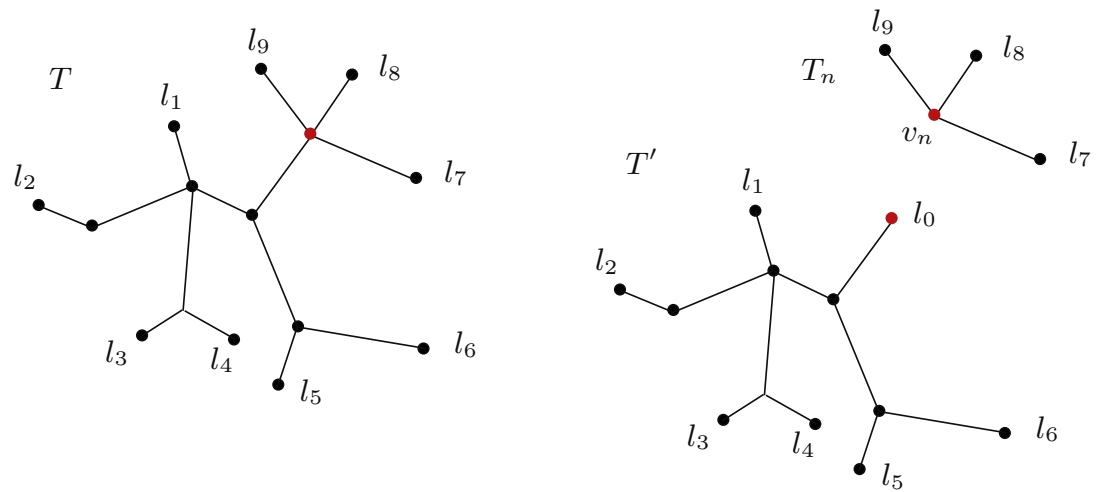

Fig. 3. A tree $T$ and the two induced subtrees $T^{\prime}$ and $T_{n}$

Let $G_{n}$ and $G^{\prime}$ be the VPT-graphs induced by all leaf-paths in $T_{n}$ and $T^{\prime}$. Both these trees have fewer inner nodes than $T$. Therefore, by induction we can assume that we have PSI-representations $P S_{n}$ of $G_{n}$ and $P S^{\prime}$ of $G^{\prime}$ as claimed in Theorem 3. We will construct a PSI-representation of $G$ using $P S_{n}$ and $P S^{\prime}$. The idea is as follows: 
1. Replace every pseudosegment of $P S^{\prime}$ representing a leaf-path ending in $l_{0}$ by a bundle of pseudosegments. This bundle stays within a narrow tube around the original pseudosegment.

2. Remove all pieces of pseudosegments from the interior of the disk $R_{0}$ and patch an appropriately transformed copy of $P S_{n}$ into $R_{0}$.

3. The crucial step is to connect the pseudosegments from the bundles through the interior of $R_{0}$ such that the induction invariants for the transformed disks of $R_{r}$ with $k \leq r \leq m$ are satisfied.

The set $\mathcal{P}$ of leaf-paths of $T$ can be partitioned into three parts. The subsets $\mathcal{P}^{\prime}$ and $\mathcal{P}_{n}$ are leaf-paths of $T^{\prime}$ or $T_{n}$ let the remaining subset be $\mathcal{P}^{*}$. The paths in $\mathcal{P}^{*}$ connect leaves $l_{i}$ and $l_{r}$ with $1 \leq i<k \leq r \leq m$, in other words they connect a leaf $l_{i}$ from $T^{\prime}$ through $v_{n}$ with a leaf in $T_{n}$. We subdivide these paths into classes $\mathcal{P}_{1}^{*}, . ., \mathcal{P}_{k-1}^{*}$ such that $\mathcal{P}_{i}^{*}$ consists of those paths from $\mathcal{P}^{*}$ which start in $l_{i}$. Each $\mathcal{P}_{i}^{*}$ consists of $\left|L_{n}\right|$ paths. In $T^{\prime}$ we have the pseudosegment $s_{i, 0}$ which leads from $l_{i}$ to $l_{0}$. Replace each such pseudosegment $s_{i, 0}$ by a bundle of $\left|L_{n}\right|$ parallel pseudosegments routed in a narrow tube around $s_{i, 0}$.

We come to the second step of the construction. Remove all pieces of pseudosegments from the interior of $R_{0}$. Recall that the representation $P S_{n}$ of $G_{n}$ from 3.2 has the property that all long pseudosegments have their endpoints on a circle $C$. Choose two arcs $A_{b}$ and $A_{t}$ on $C$ such that every segment spanned by a point in $A_{b}$ and a point in $A_{t}$ intersects each pseudosegment $s_{i, j}$ with $i \neq j$, this is possible by the choice of $C$. This partitions the circle into four arcs which will be called $A_{b}, A_{l}, A_{t}, A_{r}$ in clockwise order. The choice of $A_{b}$ and $A_{t}$ implies that each pseudosegment touching $C$ has one endpoint in $A_{l}$ and the other in $A_{r}$.

Map the interior of $C$ with an homeomorphism $h$ into a wide rectangular box $\Gamma$ such that $A_{t}$ and $A_{b}$ are mapped to the top and bottom sides of the box, $A_{l}$ is the left side and $A_{r}$ the right side. This makes the images of all long pseudosegments traverse the box from left to right. We may also require that the homomorphism maps the disks $R_{r}$ to disks and arranges them in a nice left to right order in the box, Figure 4 shows an example. The figure was generated by sweeping the representation from Figure 2 and converting the sweep into a wiring diagram (the diametrical segments $s_{r, r}$ have been re-attached horizontally).

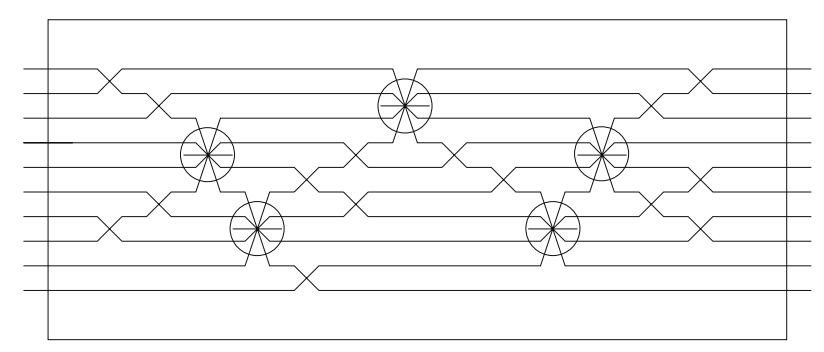

Fig. 4. A box containing a deformed copy of the representation from Figure 2 
In the box we have a left to right order of the disks $R_{r}, l_{r} \in L_{n}$. By possibly relabeling the leaves of $L_{n}$ we can assume that the disks are ordered from left to right as $R_{k}, . ., R_{m}$.

This step of the construction is completed by placing the box $\Gamma$ appropriately resized in the disk $R_{0}$ such that each of the segments $s_{i, 0}$ from the representation of $G^{\prime}$ traverses the box from bottom to top and the sides $A_{l}$ and $A_{r}$ are mapped to the boundary of $R_{0}$. The boundary of $R_{0}$ is thus partitioned into four arcs which are called $A_{l}, A_{t}^{\prime}, A_{r}, A_{b}^{\prime}$ in clockwise order. We assume that the segments $s_{i, 0}$ touch the arc $A_{b}^{\prime}$ in $P S^{\prime}$ in counterclockwise order as $s_{1,0}, . ., s_{k-1,0}$, this can be achieved by renaming the leaves appropriately.

Note that by removing everything from the interior of $R_{0}$ we have disconnected all the pseudosegments which have been inserted in bundles replacing the original pseudosegments $s_{i, 0}$. Let $B_{i}^{i n}$ be the half of the bundle of $s_{i, 0}$ which touches $A_{b}^{\prime}$ and let $B_{i}^{\text {out }}$ be the half which touches $A_{t}^{\prime}$. By the above assumption the bundles $B_{1}^{i n}, . ., B_{k-1}^{i n}$ touch $A_{b}^{\prime}$ in counterclockwise order, consequently, $B_{1}^{\text {out }}, . ., B_{k-1}^{\text {out }}$ touch $A_{t}^{\prime}$ in counterclockwise order. Within a bundle $B_{i}^{\text {in }}$ we label the segments as $s_{i, k}^{i n}, \ldots, s_{i, m}^{i n}$, again counterclockwise. The segment in $B_{i}^{\text {out }}$ which was connected to $s_{i, r}^{\text {in }}$ is labeled $s_{i, r}^{\text {out }}$ The pieces $s_{i, r}^{i n}$ and $s_{i, r}^{\text {out }}$ will be part of the pseudosegment representing the path $P_{i, r}$.

To have property (b) for the pseudosegments of a bundle we twist whichever of the bundles $B_{i}^{i n}$ or $B_{i}^{\text {out }}$ traverses $R_{i}$ within this disk $R_{i}$ thus creating a multiple intersection point. Note that they all cross $s_{i, i}$ as did $s_{i, 0}$.

Also due to (b) the pseudosegments of paths $P_{i, r}$ for fixed $r \in\{k, . ., m\}$ have to intersect in the disks $R_{r}$ inside of the box $\Gamma$. To prepare for this we take a narrow bundle of $k-1$ parallel vertical segments reaching from top to bottom of the box $\Gamma$ and intersecting the disk $R_{r}$. This bundle is twisted in the interior of $R_{r}$. Let $\check{a}_{1}^{r}, \ldots, \check{a}_{k-1}^{r}$ be the bottom endpoints of this bundle from left to right and let $\hat{a}_{1}^{r}, . ., \hat{a}_{k-1}^{r}$ be the top endpoints from right to left, due to the twist the endpoints $\check{a}_{j}^{r}$ and $\hat{a}_{j}^{r}$ belong to the same pseudosegment.

We are ready now to construct the pseudosegment $s_{i, r}$ that will represent the path $P_{i, r}$ in $T$ for $1 \leq i<k \leq r \leq m$. The first part of $s_{i, r}$ is $s_{i, r}^{i n}$, this pseudosegment is part of the bundle $B_{i}^{i n}$ and has an endpoint on $A_{b}^{\prime}$. Connect this endpoint with a straight segment to $\check{a}_{i}^{r}$, from this point there is the connection up to $\hat{a}_{i}^{r}$. This point is again connected by a straight segment to the endpoint of $s_{i, r}^{\text {out }}$ on the arc $A_{t}^{\prime}$. The last part of $s_{i, r}$ is the pseudosegment $s_{i, r}^{\text {out }}$ in the bundle $B_{i}^{\text {out }}$. The construction is illustrated in Figure 5 .

It remains to prove that the construction indeed yields a representation of $G$ as intersection graph of pseudosegments and that this representation has the properties (a) and (b) from Theorem 3. The argument is split into a series of claims.

Claim 1. There is exactly one pseudosegment $s_{i, j}$ for every pair $l_{i}, l_{j}$ of leaves of $T$.

Claim 2. The pseudosegment $s_{i, j}$ traverses $R_{i}$ and $R_{j}$ but stays disjoint from every other of the disks. 


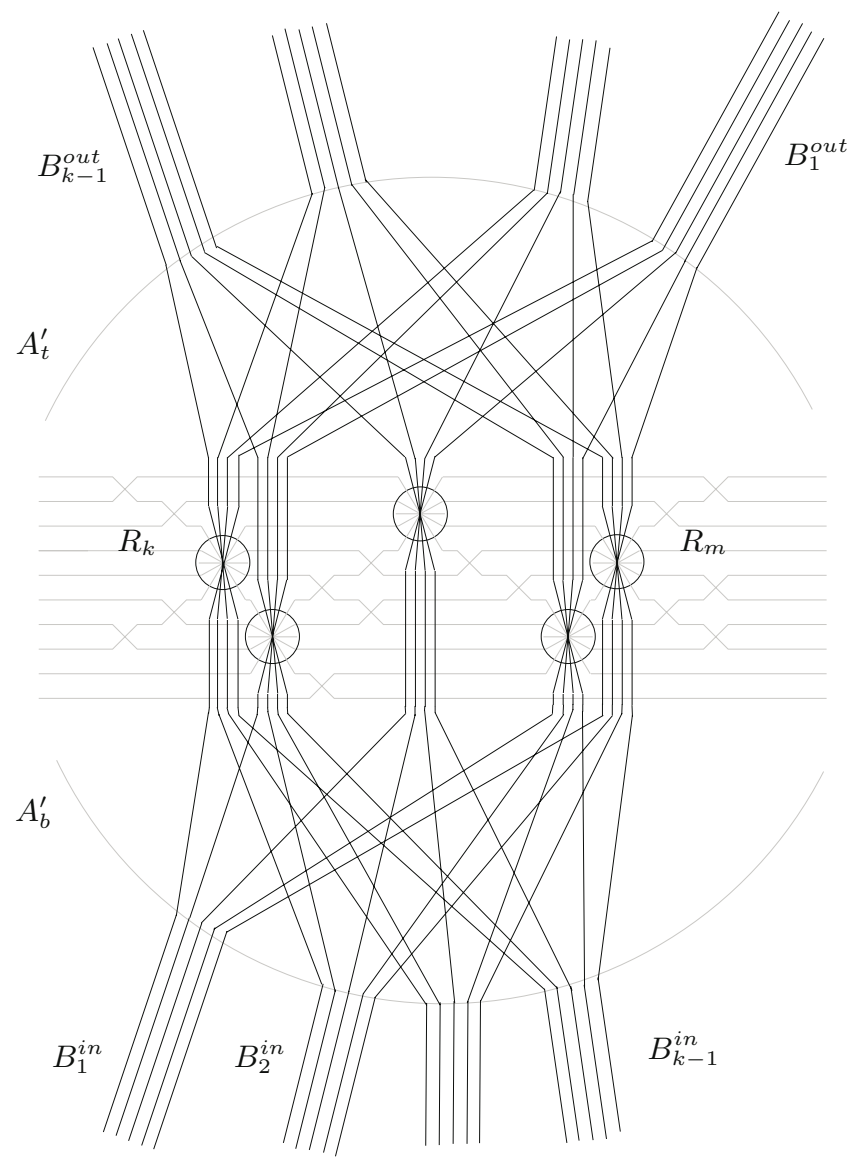

Fig. 5. The routing of pseudosegments in the disk $R_{0}$, an example

Claim 3. Any two pseudosegments intersecting the disk $R_{i}$ cross in $R_{i}$.

Claim 4. Two pseudosegments $s_{i, j}$ and $s_{i^{\prime}, j^{\prime}}$ intersect at most once, i.e., to call them pseudosegments is justified.

Claim 5. Two pseudosegments $s_{i, j}$ and $s_{i^{\prime}, j^{\prime}}$ intersect exactly if the corresponding paths $P_{i, j}$ and $P_{i^{\prime}, j^{\prime}}$ intersect in $T$.

With the verification of these claims the proof of Theorem 3 is complete. In this extended abstract we dispense with the proof.

\section{Proof of Theorem 2}

Recall the definition of the graphs $K_{n}^{3}$ from the introduction: $K_{n}^{3}$ has two groups of vertices, the set $V_{C}=\left\{v_{1}, . ., v_{n}\right\}$ induces a clique and there is an additional vertex $v_{i, j, k}$ for every triple $\{i, j, k\} \subset[n]$. These triple-vertices are adjacent only 
to the three corresponding vertices $v_{i}, v_{j}$ and $v_{k}$, hence form an independent set denoted $V_{I}$.

Assuming that there is a representation of $K_{n}^{3}$ as intersection graph of pseudosegments the set of pseudosegments can be divided into $P S_{C}$ and $P S_{I}$, i.e., the pseudosegments representing vertices from $V_{C}$ and $V_{I}$.

The pseudosegments of $P S_{C}$ form a set of pairwise crossing pseudosegments, we refer to the configuration of these pseudosegments as the arrangement $A_{n}$. The set $S=P S_{I}$ of 'small' pseudosegments has the following properties:

(i) Pseudosegments $t \neq t^{\prime}$ from $S$ are disjoint,

(ii) Every pseudosegment $t \in S$ has nonempty intersection with exactly three pseudosegments from the arrangement $A_{n}$ and no two pseudosegments $t \neq t^{\prime}$ intersect the same three pseudosegments from $A_{n}$.

The idea for the proof is to show that a set of pseudosegments with properties (i) and (ii) only has $O\left(n^{2}\right)$ elements. The theorem follows, since $|S|=\left(\begin{array}{l}n \\ 3\end{array}\right)=\Omega\left(n^{3}\right)$.

\subsection{Geometric Restriction}

Every pseudosegment $p \in A_{n}$ is cut into $n$ pieces by the $n-1$ other pseudosegments of $A_{n}$. Let $W$ be the set of all the pieces obtained from pseudosegments from $A_{n}$, note that $|W|=n^{2}$. A triple-segment $t \in S$ intersects with exactly three pieces of three different pseudosegments of $A_{n}$. Hence it has a unique middle and two outer intersections. Let $S(w)$ be the set of triple-segments with middle intersection on the piece $w \in W$. The set $S$ is partitioned as $S=\dot{U}_{w \in W} S(w)$.

Define $G_{p}=\left(W, E_{p}\right)$ as the simple graph where two pieces $w, w^{\prime}$ are adjacent if and only if there exists a triple-segment $t \in S$ such that $t$ has its middle intersection on $w$ and an outer intersection on $w^{\prime}$.

Lemma 2. $G_{p}=\left(W, E_{p}\right)$ is planar.

Proof. A planar embedding of $G_{p}$ is induced by $A_{n}$ and $S$. Contract all pieces from pseudosegments in $A_{n}$, the contracted pieces represent the vertices of $G_{p}$.
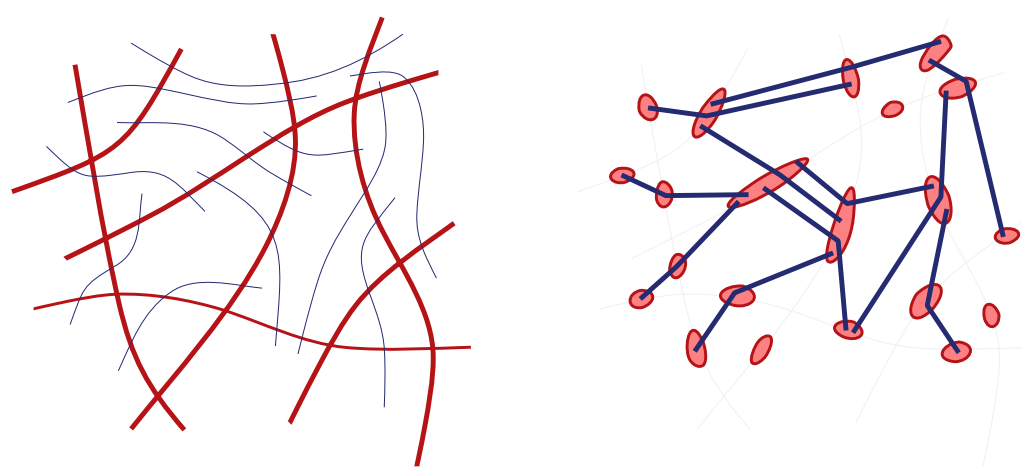

Fig. 6. A part of $A_{n}$ with some triple-segments and the edges induced by them of $G_{p}$ 
The pseudosegments in $S$ are pairwise non-crossing, this property is maintained during contraction of pieces, see Figure 6. If $t \in S$ has middle piece $w$ and outer pieces $w^{\prime}$ and $w^{\prime \prime}$, then $t$ contributes the two edges $\left(w, w^{\prime}\right)$ and $\left(w, w^{\prime \prime}\right)$. Hence, the multigraph obtained through these contractions is planar and its underlying simple graph is indeed $G_{p}$.

Let $N(w)$ be the set of neighbors of $w \in W$ in $G_{p}$ and let $d_{G_{p}}(w)=|N(w)|$.

Lemma 3. The size of a set $S(w)$ of triple-segments with middle piece $w$ is bounded by $d_{G_{p}}(w)-1$ for every $w \in W$.
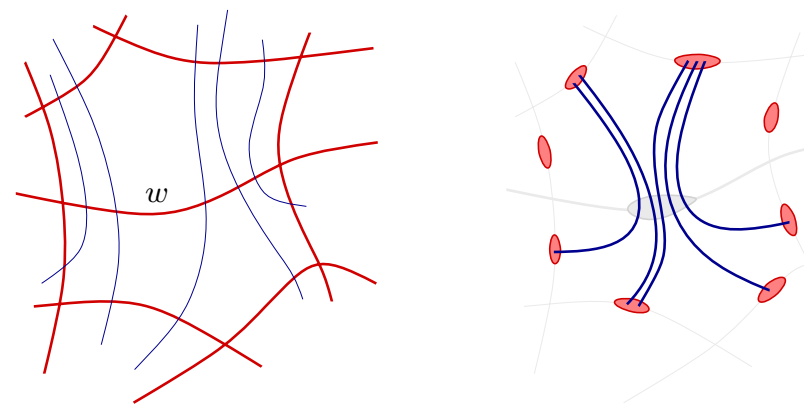

Fig. 7. The planar graph $N G_{w}$ induced by the triple-segments with middle intersection

Proof. Define the neighborhood graph $N G_{w}=\left(N(w), E_{w}\right)$ for every $w \in W$ where two vertices $u, u^{\prime}$ are adjacent, if and only if there is a short pseudosegment $t \in S(w)$ with $u$ and $u^{\prime}$ as outer pieces. The number of edges of $N G_{w}$ equals the number of triple-segments in $S(w)$. The idea is to contract just the pieces corresponding to elements of $N(w)$ to points. The triple-segments in $S(w)$ together with the vertices obtained by contraction form a planar graph, see Figure 7. Note that the resulting graph is not a multigraph, since a multiple edge would correspond to a pair of triple-segments intersecting the same three pieces of $A_{n}$. We will show that $N G_{w}$ is acyclic, hence a forest. This implies the statement of Lemma 3 .

Assume there was a cycle $C$ in $N G_{w}$. Label its vertices $w_{1}, . ., w_{k} \in N(w)$ such that $w_{k} w_{1}$ and $w_{i} w_{i+1}$ with $1 \leq i<k$ are the edges of $C$. Let $t_{i}$ be the triple-segment defining edge $w_{i} w_{i+1}$. Recall that $t_{i}$ intersects $w_{i}, w$ and $w_{i+1}$. A cycle in $N G_{w}$ corresponds to a simple closed curve in the PSI-representation as follows: Denote the part of $w_{i}$ connecting its crossings with $t_{i}$ and $t_{i-1}$ by $a_{i}$. As $w_{i} \in W$ is a piece and $t_{j} \in S(w) \subseteq S$, the set of pieces and triple-segments contributing to $C$ as vertices or edges does not induce more crossings than the pairs $\left(w_{i}, t_{j}\right)$ with $j \in\{i-1, i\}$. Ignoring the pending ends of $t_{i}$, the union of the $a_{i}$ and $t_{i}, i \in\{1, \ldots, k\}$ corresponds to a simple closed curve $\Gamma$ within the PSI-representation. The curve $\Gamma$ is the concatenation of $a_{i}$ and $t_{i}$ in the order $a_{1}, t_{1}, a_{2}, . ., t_{k-1}, a_{k}, t_{k}$, see Figure 8 . 


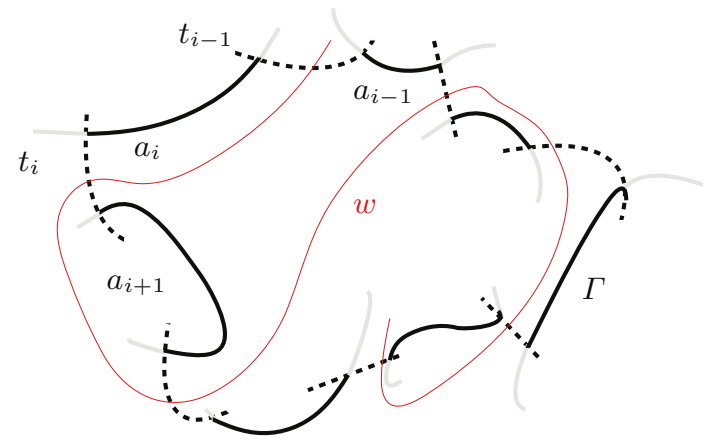

Fig. 8. A chain of segments corresponding to a cycle in $N G_{w}$

Note that $k \geq 3$, as $N G_{w}$ is not a multigraph. Choose three triple-segments $t_{j}, t_{i}, t_{l}$ from $\Gamma$ such that they intersect $w$ in this order and no other triplesegment of $C$ crosses $w$ between them. We will identify a simple closed curve $\gamma$ separating $w_{i}$ and $w_{i+1}$. Even more, the complete pseudosegments $p$ and $p^{\prime}$ containing $w_{i}$ respectively $w_{i+1}$ will be separated by $\gamma$. This is a contradiction to the fact that they both belong to the set $A_{n}$ of pairwise intersecting pseudosegments.

Such a curve $\gamma$ can be described as follows: By possibly shifting the indices of $w_{i}$ along $C$, we can assume $i<j<l$. Denote the part of $w$ between its intersections with $t_{j}$ and $t_{l}$ by $w_{j, l}$. Be $P_{j+1, l}$ the subpath of $C \backslash w_{i}$ connecting $w_{j+1}$ and $w_{l}$, denote the corresponding part of $\Gamma$ by $\Gamma_{j+1, l}$. Connect $w_{j, l}$ to $\Gamma_{j+1, l}$ at $t_{j}$ and $t_{l}$. This gives a simple closed curve $\gamma$. The curve $\gamma$ consists of an arc of $\Gamma$, the arc $w_{j, l}$ of $w$ and parts of $t_{j}$ and $t_{l}$. It follows that $\gamma$ can not be crossed by a pseudosegment from $A_{n}$. Recall that by definition of PSI-representations an intersection of pseudosegments implies, that they cross. Applied to the intersection of $t_{i}$ and $w_{j, l} \subset w$ this has the consequence that $w_{i}$ and $w_{i+1}$ are on

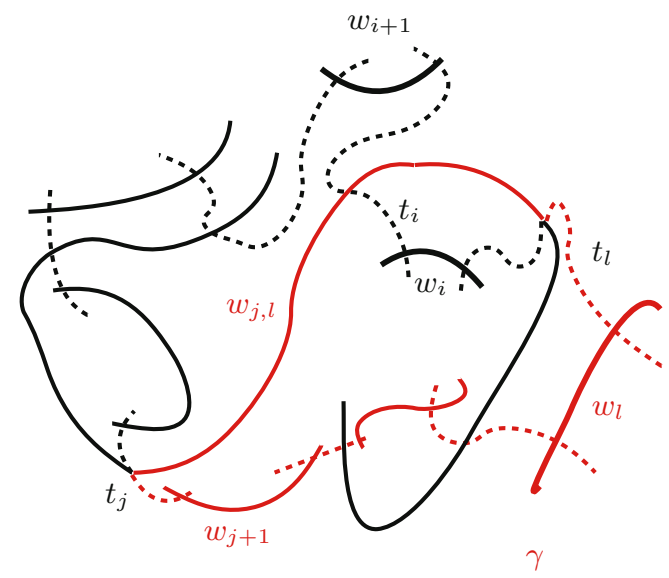

Fig. 9. Pieces $w_{i}$ and $w_{i+1}$ are separated by $\gamma$ 
different sides of $\gamma$, see Figure 9, As shown before this implies that the complete pseudosegments $p$ containing $w_{i}$ and $p^{\prime}$ containing $w_{i+1}$ have to lie on different sides of $\gamma$, hence they can not intersect, a contradiction to the choice of $A_{n}$. Thus $N G_{w}$ is acyclic and $E_{w}$ is bounded by $d_{G_{p}}(w)-1$.

In addition to the result of Lemma 3 we know

- $|W|=n^{2}$,

- $\sum_{w \in W} d_{G_{p}}(w)=2\left|E_{p}\right|<6|W|$.

This implies $|S|=\sum_{w \in W}|S(w)| \leq \sum_{w \in W}\left(d_{G_{p}}(w)-1\right)<6|W|=6 n^{2}$.

Since $\left(\begin{array}{l}n \\ 3\end{array}\right)>6 n^{2}$ for all $n \geq 39$ we conclude that $K_{n}^{3}$ does not belong to PSI for $n \geq 39$. This completes the proof of Theorem 2 ,

\section{Conclusions and Further Questions}

We have investigated the containment relation between the classes of PSI-graphs and of chordal graphs. This may stimulate investigations concerning the relation between PSI-graphs and other classes. Of course the main open problem in the area remains the question whether all planar graphs are PSI-graphs.

\section{References}

1. M.Bodirsky, C.Dangelmayr and J. Kára: Representing Series-parallel Graphs as Intersection Graphs of Line Segments in Three Directions, submitted.

2. N. de Castro, F. J. Cobos, J. C. Dana and A. Márquez: Triangle-Free Planar Graphs as Segment Intersection Graphs, Journal of Graph Algorithms and Applications, Volume 6, pp. 7-26, 2002.

3. I. Ben-Arroyo Hartman, I. Newman and R. Ziv: On grid intersection graphs, Discrete Math., 87, pp. 41-52, 1991.

4. H. de Fraysseix, P.O. de Mendez and J. Pach: Representation of planar graphs by segments. Colloquia Mathematica Societatis János Bolyai, Intuitive Geometry, Szeged (Hungary), 1991.

5. H.de Fraysseix and P.O. de Mendez: Contact and Intersection Representations, GD 2004, Lecture Notes in Computer Science, Volume 3383, pp. 217-227, 2005.

6. F. Gavril, A recognition algorithm for the intersection graphs of paths in trees Discrete Math. Volume 23, pp. 211-227, 1978.

7. J. Kratochvil: String Graphs II: Recognizing String Graphs is NP-hard, Journal of Comb. Theory,Ser. B 52,No. 1, pp. 67-78, 1991.

8. J. Kratochvil: A special planar satisfiability problem and a consequence of its NP-completeness, Discrete Applied Mathematics, 52, pp. 233-252 , 1994.

9. J. Kratochvil and J. Matoušek: Intersection graphs of Segments, Journal of Comb. Theory, Ser. B 62, pp. 289-315, 1994.

10. C. Monma and V. K. Wei, Intersection graphs of paths in a tree, Journal of Comb. Theory, Ser. B 41, pp. 141-181, 1986.

11. M. Schaefer, E. Sedgwick and D. Stefanovic: Recognizing string graphs in NP, Journal of Comput. Syst.Sci., No. 2, pp. 365-380, 2003.

12. E. R. Scheinerman: Intersection classes and multiple intersection parameters of graphs, $\mathrm{PhD}$ thesis, Princeton University 1984. 